\section{Ein zutiefst menschliches Geschäft}

\section{Über das Erfolgskonzept des Pflegeheimanbieters SeneCura}

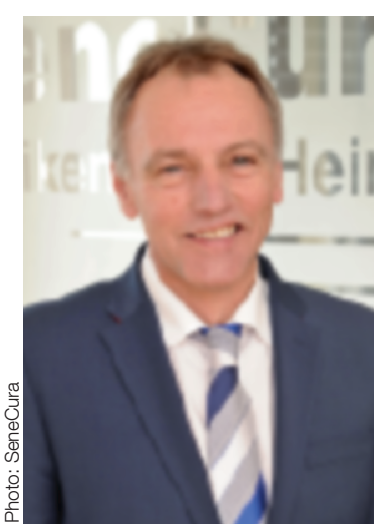

Anton Kellner

Geschäftsführer der SeneCura Gruppe

Bei SeneCura, dem größten privaten Pflegeheimbetreiber Österreichs, steht der Mensch im Mittelpunkt. Anton Kellner, Geschäftsführer der SeneCura Gruppe, berichtet anlässlich des 20 -jährigen $B e-$ stehens von procare über das Erfolgskonzept von SeneCura, einem langjährigen Kooperationspartner von procare, und über die Pläne für die Zukunft.

Die SeneCura Gruppe ist mit etwa 70 Pflegeeinrichtungen der größte private Pflegeheimbetreiber in Österreich. Was ist das Erfolgsrezept von SeneCura? KELLNER: Seit der Unternehmensgründung im Jahr 1998 ist es uns wichtig, unsere Geschäftstätigkeit an den Bedürfnissen unserer Bewohner auszurichten. Es galt und gilt für uns, mit unseren Angeboten ihre Lebensqualität zu steigern und Versorgungslücken mit alternativen Betreuungsangeboten zu schließen. Getreu unseres Mottos „Näher am Menschen“ steht für uns immer der Mensch im Mittelpunkt, mit der individuellen Pflege und Betreuung, die er sich wünscht und braucht. Danach richten wir unsere Arbeit jeden Tag aus, denn unser Geschäft ist ein zutiefst menschliches.

Was ist das Besondere an den Betreuungseinrichtungen von SeneCura? KELLNER: Wir bieten individuelle Betreuung und Pflege, ganz nach Wunsch und
Bedarf. Und das ist nicht alles: Spezialangebote wie die rehabilitative Übergangspflege, das Mobilitätsprogramm „Fit \& Beweglich 77+" oder auch das Projekt "Schmerzfreies Pflegeheim“ unterscheiden uns vom Mitbewerb. Wohlfühlen bedeutet in unseren Augen mehr als nur gesund zu sein: Deshalb bringen wir Freude in den Alltag - durch regelmäßige Feste, Feiern und Ausflüge, einen Urlaubsaustausch zwischen den Häusern und ein buntes Freizeitprogramm mit BastelnachBacken. Auch die Einbindung der Angehörigen sowie ortsansässiger Schulen, Vereine und Kindergärten ist uns sehr wichtig, denn wir sehen uns als integrativer Bestandteil der Gemeinden, in denen wir tätig sind. Unsere zahlreichen ehrenamtlichen Helfer sind für uns eine sehr große Unterstützung und sie bereichern unglaublich den Pflegealltag sowohl für unsere Bewohner als auch für unser Team. Vielen Dank an alle unsere Ehrenamtlichen!

Unter der Marke „OptimaMed“ baut die SeneCura Gruppe auch den Rehabereich weiter aus. Was sind hier die aktuellen Projekte?

KELLNER: Um den Gesundheitsbereich stärker von der Pflege zu trennen, bekam er 2014 einen eigenen Markenauftritt: Unter dem Namen OptimaMed werden alle medizinischen und rehabilitativen Angebote der SeneCura Gruppe zusammengefasst. Bereits 2012 haben wir mit der Eröffnung des Neurologischen Rehabilitationszentrums Kittsee unser Angebot um medizinische Einrichtungen erweitert. Im März diesen Jahres eröffneten wir ein Dialysezentrum in Frauenkirchen. Außerdem fand im Winter 2014 der Spatenstich für das neue physikalische Ambulatorium Kittsee statt und im steirischen Wildbad wird derzeit ein Gesundheitskompetenzzentrum mit zahlreichen rehabilitativen und physikalisch-medizinischen Angeboten ausgebaut.

Pflege ist ein Berufsfeld mit Zukunft und ein wichtiger Wirtschaftsfaktor. Wie können die Gemeinden, in denen Sie tätig sind, von SeneCura profitieren? mittagen oder gemeinsamem Kochen und
KELLNER: Als Teil des gesellschaftlichen Lebens der Gemeinde legt SeneCura großen Wert darauf, dass wir mit unseren Einrichtungen einen Beitrag zur Erhaltung und Förderung der regionalen Wirtschaft leisten. So werden selbstverständlich ortsansässige Lieferanten und Dienstleister als Partner in den Tagesablauf der Häuser integriert, und beispielsweise als Lebensmittelversorger der großteils hauseigenen Küchen beauftragt. Darüber hinaus bieten die Betreuungsreinrichtungen der lokalen Bevölkerung attraktive und sichere Arbeitsplätze in den Bereichen Pflege, Facility Management und anderen Dienstleistungen.

Spielt das Thema Nachhaltigkeit bei SeneCura eine Rolle?

KELLNER: Ja, absolut! Nachhaltigkeit ist eine strategische Zielsetzung von SeneCura, deshalb achten wir auf einen effizienten Einsatz aller Ressourcen. Wir prüfen vor allen unternehmerischen Entscheidungen nicht nur die finanziellen Konsequenzen, sondern stellen gleichzeitig auch die soziale und ökologische Nachhaltigkeit in den Mittelpunkt. Dass unser Engagement Früchte trägt, beweist der erste Platz in der Kategorie „Most Socially/Environmentally Responsible Company of the Year", den SeneCura bei den renommierten Best in Biz Awards für den Raum EMEA 2014 erreicht hat.

Wie wird die Zukunft aussehen - bei SeneCura und der Branche allgemein? KELLNER: Die Lebenserwartung steigt kontinuierlich. Gleichzeitig gehen die $\mathrm{Ge}$ burtenraten stetig zurück. Die logische Konsequenz: Viele ältere Menschen können nicht mehr wie früher von der eigenen Familie gepflegt werden, der externe Pflegebedarf steigt rasant an. Dabei sind heute vor allem mobile Pflegeformen sehr beliebt. Diesem Anspruch kommen wir mit unserer 24- Stunden-Betreuung nach. Aber auch im Bereich der stationären Pflege wird der Bedarf weiter steigen: Bis 2020 werden etwa 15.000 zusätzliche Plätze benötigt. Bei SeneCura sind wir bemüht, unsere Standorte kontinuierlich auszubauen und so eine flächendeckende Betreuung zu garantieren. Daher werden wir auch in den kommenden Jahren wieder einige neue Häuser eröffnen - nicht nur in Österreich, sondern auch in der Tschechischen Republik. Denn auch hier steigt die Nachfrage an Betreuungsleistungen und Einrichtungen für Betreutes Wohnen weiter. 